\title{
Consenso sobre la monitorización del recién nacido internado. Parte 2: Monitorización según los niveles de complejidad
}

Consensus in hospitalized newborn monitoring. Part 2: Monitoring according to levels of complexity

Dra. Gisela Salas ${ }^{a}$, Dra. Daniela Satragno ${ }^{b}$, Dra. Patricia Bellani ${ }^{a}$, Lic. Ana Quirogac, Dr. Gastón Pérez ${ }^{d}$, Lic. Norma Erpen ${ }^{a}$, Enf. Gustavo Villalba ${ }^{d}$, Dra. Laura Cortea ${ }^{e}$, Lic. Graciela Centenof, Dr. Raúl Musantes, Dra. Graciela Aracama ${ }^{h}$, Dra. Marta Álvarez', Lic. Claudia Rearte $i$, Dra. Mónica Ganduglia y Dra. Diana Fariña ${ }^{a}$

http:/ /dx.doi.org/10.5546/aap.2013.440

\section{Tensión arterial invasiva}

La monitorización de la tensión arterial invasiva (TAI) continúa siendo el patrón de referencia para la medición de la tensión arterial en los pacientes críticos. ${ }^{1,2}$

Funcionamiento: la medición directa de la TAI se efectúa a través de un catéter introducido en una vía arterial y acoplado a un transductor de presión. El torrente circulatorio ejerce fuerza contra un diafragma siliconado que la registra como una señal eléctrica y luego traduce la presión leída en milímetros de mercurio $(\mathrm{mm} \mathrm{Hg})$, registrando valores sistólicos, diastólicos y medios ${ }^{3}$ (Figura 1).

Indicaciones:

- Pacientes prematuros en asistencia ventilatoria hasta su estabilización.

- Pacientes con requerimientos de inotrópicos intravenosos. ${ }^{1}$

- Pacientes con requerimiento de altos parámetros de asistencia ventilatoria mecánica (AVM), ventilación de alta frecuencia (VAF) y óxido nítrico inhalado.

- Pacientes sometidos a cirugía mayor o procedimientos complejos. ${ }^{3}$

Dra. Gisela Salas: gsalasenator@gmail. com

Fuentes de financiación: Ninguna.

Conflicto de intereses: Ninguno que declarar.

Recibido:

Aceptado:
- En caso de colocar catéteres en la arteria radial, es necesario realizar la prueba de Allen para comprobar la presencia de circulación colateral por la arteria cubital. El riesgo de lesión vascular secundaria a la colocación de arteria radial en $\mathrm{RN}$ pequeños es de un $5 \%$. Es fundamental rotularla para evitar confundirla con un acceso venoso. ${ }^{4}$

- La medida del catéter debe ser adecuada al peso del paciente. A menor tamaño del catéter, aumentan los errores de la monitorización y la posibilidad de trombosis.

- Pérdida de la onda del pulso: se recomienda lavar el catéter con solución heparinizada y calibrar a cero, con el transductor ubicado a nivel de las aurículas del paciente; si el transductor de presión se encuentra alto medirá menor presión, y viceversa.

Figura 1. Onda de tensión arterial invasiva

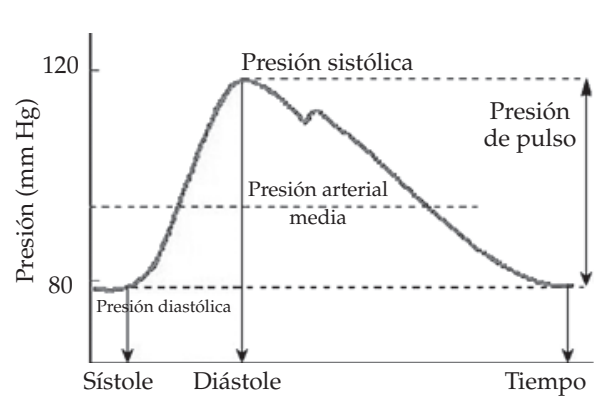


- Controlar frecuentemente la presencia de aire o sangre en el sistema.

- El transductor es de uso individual y descartable.

- Si el catéter es de doble luz permitirá tomar muestras de sangre sin suspender la medición de la TA.

- Se deben controlar las llaves de tres vías, el ajuste de sus roscas y la ausencia de rotura para evitar las pérdidas.

- El sistema se debe purgar antes de la conexión al paciente y controlar que no haya restos de sangre ni aire.

- Nunca hay que olvidarse de colocar las alarmas. La hipotensión arterial generalmente se acompaña de alteración en otros parámetros (FC, saturación), así como del estado clínico del paciente (relleno capilar, perfusión, $\mathrm{pH}$ sanguíneo, ácido láctico, etc.); por lo tanto, los parámetros monitorizados deben evaluarse en el contexto global del paciente antes de tomar decisiones (Tabla 1).

\section{Complicaciones}

Son inherentes a la colocación de un catéter arterial.

\section{Presión venosa central}

La presión venosa central (PVC) no es de uso habitual en neonatología. Sin embargo, en el paciente hemodinámicamente inestable, es útil para evitar la sobredosificación de volumen que puede provocar disfunción miocárdica. Los valores bajos de PVC en el RN pueden ser normales.

Técnicamente, el transductor es el mismo que el usado para el control de la TA invasiva.

Se considera una PVC alta si es mayor o igual a $8 \mathrm{~mm} \mathrm{Hg}{ }^{3}$

\section{Complicaciones}

Obstrucción, perforación miocárdica, taponamiento cardíaco, arritmias, tromboembolia, flebitis, endocarditis, etc.

\section{Catéter arterial umbilical}

Todo paciente crítico debe contar con un catéter arterial umbilical para su correcta monitorización.

\section{Indicaciones:}

- Medición frecuente de gases en sangre (prematuros). ${ }^{5}$

- Medición continua de la tensión arterial invasiva.

TABLA 1. Resolución de problemas en la medición de la tensión arterial invasiva

\begin{tabular}{|c|c|c|c|}
\hline Problema & Causa & Prevención & Tratamiento \\
\hline \multirow[t]{3}{*}{$\begin{array}{l}\text { Desaparición del } \\
\text { registro de onda }\end{array}$} & $\begin{array}{l}\text { El catéter está contra la pared } \\
\text { del vaso }\end{array}$ & Inevitable & $\begin{array}{l}\text { Reposicionar el catéter } \\
\text { hasta observar la onda }\end{array}$ \\
\hline & $\begin{array}{l}\text { Oclusión parcial del catéter por } \\
\text { coágulos }\end{array}$ & $\begin{array}{l}\text { Infusión continua de solución } \\
\text { heparinizada ( } 0,5 \text { a } 1 \mathrm{U} / \mathrm{mL}) \text {. } \\
\text { Evitar los bolos de solución } \\
\text { heparinizada }\end{array}$ & $\begin{array}{l}\text { Aspirar los coágulos } \\
\text { con jeringa e infusión } \\
\text { continua de solución } \\
\text { heparinizada }\end{array}$ \\
\hline & $\begin{array}{l}\text { Presencia de sangre o coágulos } \\
\text { en el sistema }\end{array}$ & $\begin{array}{l}\text { Infundir cuidadosamente, retirar } \\
\text { la sangre del sistema }\end{array}$ & $\begin{array}{l}\text { Aspirar los coágulos, } \\
\text { infundir solución } \\
\text { heparinizada o cambiar } \\
\text { el sistema (transductor, } \\
\text { llaves de tres vías) }\end{array}$ \\
\hline \multirow{3}{*}{$\begin{array}{l}\text { Mediciones } \\
\text { anormalmente } \\
\text { altas o bajas de la } \\
\text { presión }\end{array}$} & $\begin{array}{l}\text { Transductor colocado por } \\
\text { encima o debajo del paciente }\end{array}$ & $\begin{array}{l}\text { Mantener al paciente y el sistema } \\
\text { en la misma posición }\end{array}$ & $\begin{array}{l}\text { Colocar el transductor a } \\
\text { la altura de la aurícula } \\
\text { derecha }\end{array}$ \\
\hline & $\begin{array}{l}\text { Pérdida de las conexiones del } \\
\text { transductor }\end{array}$ & $\begin{array}{l}\text { Asegurar el transductor } \\
\text { firmemente, usar llaves de tres } \\
\text { vías descartables }\end{array}$ & $\begin{array}{l}\text { Ajustar las uniones del } \\
\text { transductor con el domo } \\
\text { y la conexión de todas las } \\
\text { llaves }\end{array}$ \\
\hline & Transductor dañado & $\begin{array}{l}\text { Evitar la excesiva presión negativa } \\
\text { al aspirar }\end{array}$ & Reemplazar el transductor \\
\hline $\begin{array}{l}\text { Pérdida de la onda } \\
\text { a pesar del lavado } \\
\text { del catéter }\end{array}$ & Burbujas de aire en el sistema & $\begin{array}{l}\text { Manipular el sistema con cuidado, } \\
\text { detectar la presencia de burbujas }\end{array}$ & $\begin{array}{l}\text { Aspirar la burbuja o } \\
\text { cambiar el sistema. NO } \\
\text { INFUNDIR hasta retirar } \\
\text { el aire }\end{array}$ \\
\hline No se observa onda & $\begin{array}{l}\text { Transductor cerrado o falta de } \\
\text { calibración a cero }\end{array}$ & $\begin{array}{l}\text { Seguir en forma sistemática los } \\
\text { pasos de rutina }\end{array}$ & $\begin{array}{l}\text { Revisar el sistema, las } \\
\text { llaves de tres vías y las } \\
\text { conexiones al monitor }\end{array}$ \\
\hline
\end{tabular}


- Exanguinotransfusión.

- Reanimación cardiopulmonar (el catéter venoso umbilical es de primera elección).

- Infusiones.

Contraindicaciones:

- Evidencia de compromiso vascular local en los miembros inferiores.

- Abdomen agudo.

- Onfalitis.

- Onfalocele.

Claves para un correcto empleo del catéter arterial umbilical:

- El tamaño del catéter depende del peso del paciente: 5 French (Fr) para mayores de $1200 \mathrm{~g}$ y 3,5 Fr para menores de ese peso.

- Los catéteres arteriales umbilicales colocados en una posición alta (entre la sexta y la novena vértebra torácica) ocasionan menores complicaciones. No hay evidencias que apoyen el uso de catéteres arteriales umbilicales de ubicación baja. Es imprescindible el control radiográfico. Se debe examinar en forma frecuente la coloración en la punta de los dedos. ${ }^{6}$

- Los catéteres con orificio terminal se asocian a una incidencia menor de trombosis en comparación con los de orificio lateral.

- Los catéteres de poliuretano y silicona ocasionan menos complicaciones que los de policloruro de vinilo (PVC) ${ }^{4}$ No se deben utilizar sondas de alimentación para canalizar el cordón umbilical.

- La utilización de catéteres arteriales umbilicales de doble luz permite la toma de muestra y la medición de la TAI simultáneamente.

- Evitar infundir con jeringa soluciones a alta presión, ya que podría provocar la rotura del catéter o tromboembolia.

- El retiro del catéter arterial se debe realizar en forma lenta $(1 \mathrm{~cm} / \mathrm{min})$.

- Velocidad de infusión: siempre lento para evitar aumentos rápidos en el flujo sanguíneo cerebral.

Complicaciones:

- Espasmo vascular: se evidencia por el cambio de coloración transitorio de los miembros, que revierte rápidamente. La coloración pálida del miembro es indicación de retiro inmediato del catéter.

- Trombosis en el catéter: produce la pérdida de la onda del pulso y, si la oclusión es total, la imposibilidad de tomar muestras de sangre.

- Trombosis aórtica: el riesgo aumenta con el tiempo de colocación del catéter.
- Por mal posicionamiento del catéter: perforación del vaso, hipoglucemia por posicionamiento en el tronco celíaco, falso aneurisma, dirección incorrecta hacia los miembros inferiores.

- Hemorragia: por desconexión del sistema cerrado.

- Enterocolitis necrosante: frente a signos clínicos de enteritis se debe retirar el catéter.

La colocación del catéter puede presentar complicaciones serias, infecciosas y mecánicas; sin embargo, el RN críticamente enfermo debe tener la monitorización adecuada a la complejidad de su estado. El catéter arterial se retira cuando la situación clínica se estabiliza. ${ }^{4,7}$

\section{Catéter venoso umbilical}

El catéter venoso umbilical es una vía de acceso central empleada en general en los primeros 7 días de vida. Debe retirarse cuando el paciente se encuentre en condiciones de manejarse con una vía venosa percutánea considerada de larga permanencia. ${ }^{4}$

Existen catéteres de una, dos y tres luces para la administración de fármacos incompatibles entre sí.

\section{Indicaciones:}

- RN crítico con requerimiento de expansiones, correcciones o transfusiones frecuentes.

- Acceso venoso rápido en la reanimación del $\mathrm{RN}$ en la sala de partos.

- Infusión de solución hidroelectrolítica o nutrición parenteral intravenosa.

- Administración de medicación en infusión continua.

- Monitorización de la presión venosa central (PVC).

Contraindicaciones:

- Onfalitis.

- Onfalocele.

- Enterocolitis necrosante.

- Peritonitis.

\section{Claves para un correcto empleo del catéter} venoso umbilical:

- Utilizar catéteres $5 \mathrm{Fr}$ para $<3,5 \mathrm{~kg}$ y de 5 a 8 Fr para $>3,5 \mathrm{~kg}$ de peso. ${ }^{4}$

- Los catéteres utilizados para exanguinotransfusión deben tener orificios laterales y deben retirarse después del procedimiento.

- Solo en la emergencia se puede colocar el catéter venoso umbilical bajo.

Complicaciones:

- Trombosis. $^{8}$

- Derrame pericárdico. 
- Infección.

- Rotura del catéter. ${ }^{4}$

\section{Monitorización transcutánea de $\mathrm{CO}_{2}$}

Las medición de $\mathrm{CO}_{2}$ de forma transcutánea ( $\mathrm{TC} \mathrm{CO}_{2}$ ) provee información continua y no invasiva del $\mathrm{CO}_{2}$. Permite monitorizar sus valores a través del tiempo y evaluar su tendencia. Si bien el método de referencia para la medición del $\mathrm{CO}_{2}$ es la gasometría arterial, esta provee solo una medición estática y transversal de un proceso clínico, además de requerir la extracción de sangre, entre otras dificultades. ${ }^{9,10}$

La TC $\mathrm{CO}_{2}$ brinda un valor estimado de la presión parcial de dióxido de carbono. Los sensores transcutáneos de $\mathrm{CO}_{2}$ calientan la piel hasta una temperatura de alrededor de $40-42^{\circ} \mathrm{C}$. Esto genera vasodilatación del lecho capilar, lo que facilita la difusión de $\mathrm{CO}_{2}$ desde la luz del capilar a la membrana del $\mathrm{TC} \mathrm{CO}_{2}$. La difusión del $\mathrm{CO}_{2}$ a la membrana cambia el $\mathrm{pH}$ de la solución electrolítica contenida en el electrodo y el valor de $\mathrm{pH}$ es convertido a un valor de $\mathrm{PCO}_{2}$ que es corregido por temperatura. ${ }^{11}$

\section{Indicaciones:}

- Estimar la $\mathrm{PCO}_{2}$ del paciente.

- Monitorizar la respuesta a las maniobras ventilatorias terapéuticas, especialmente en los pacientes con ventilación de alta frecuencia, que pueden sufrir cambios rápidos en el $\mathrm{CO}_{2}$

- Estabilización y monitorización durante el transporte.

- Reducir la frecuencia de la extracción de sangre para realizar EAB, especialmente con apoyo ventilatorio crónico.

- Determinar las tendencias del $\mathrm{CO}_{2}$ en un paciente a través del tiempo.

\section{Contraindicaciones:}

- Enfermedades extensas de la piel, epidermólisis, estafilodermia, etc.

- Contraindicaciones relativas: acidosis grave, mala perfusión periférica.

Claves para una correcta monitorización:

- Respetar el tiempo de calibración del equipo.

- Colocar el sensor en áreas con buen flujo sanguíneo como la zona subxifoidea o los flancos.

- Para evitar quemaduras en la piel, se debe cambiar la ubicación del sensor según la recomendación del fabricante, habitualmente cada 4 a 6 horas.

- Recordar que la velocidad de respuesta es lenta, el tiempo de respuesta promedio es de 60 a 90 segundos.

\section{Complicaciones:}

Lesiones de la piel, entre ellas, quemaduras.

\section{Capnografía}

El monitoreo continuo y no invasivo de $\mathrm{CO}_{2}$ $\left(\mathrm{EtCO}_{2}\right)$ en la terapia intensiva neonatal puede poner en evidencia los episodios de hipocapnia e hipercapnia, y evitar las extracciones reiteradas de sangre. El análisis y registro continuo de la concentración de $\mathrm{CO}_{2}$ en los gases respiratorios se denomina capnografía. El equipo que mide y muestra valores numéricos de $\mathrm{CO}_{2}$ respiración a respiración se denomina capnómetro, mientras que el que muestra la curva de $\mathrm{CO}_{2}$ durante el ciclo respiratorio se denomina capnógrafo. La mayoría de los capnógrafos emplean tecnología infrarroja basada en la absorción infrarroja de $\mathrm{CO}_{2}$ y pueden tener dos tipos de analizadores: aquellos cuyo sensor se ubica en línea con el tubo endotraqueal y otros de tipo lateral que cuentan con un adaptador de bajo espacio muerto. ${ }^{12,13}$

\section{Indicaciones:}

- Evaluación del $\mathrm{CO}_{2}$ exhalado.

- Identificación de la presencia de apneas y obstrucción de la vía aérea.

- Monitorización de la enfermedad pulmonar grave.

- Evaluación de la respuesta al tratamiento, particularmente cuando se intenta modificar el espacio muerto o mejorar las alteraciones de la ventilación-perfusión.

La ventilación del espacio muerto se puede calcular con la siguiente fórmula:

$$
\mathrm{Vd} / \mathrm{Vt}=\frac{\mathrm{PaCO}_{2}-\mathrm{ETCO}_{2}}{\mathrm{ETCO}_{2}}
$$

donde:

Vd: Ventilación espacio muerto.

Vt: Volumen corriente (tidal).

El valor normal es 0,3 (30\% del volumen corriente corresponde al espacio muerto).

\section{Claves para el correcto monitoreo:}

- Cuando el espacio muerto es grande se afecta la medición de $\mathrm{EtCO}_{2}$, la diferencia entre el $\mathrm{EtCO}_{2}$ y la $\mathrm{PaCO}_{2}$ aumenta cuando el espacio muerto aumenta. $\mathrm{El} \mathrm{CO}_{2}$ espirado cae y el Vd/ Vt será mayor.

- Recordar que el adaptador de $\mathrm{EtCO}_{2}$ puede agregar resistencia y espacio muerto al circuito.

- La correlación de las mediciones de $\mathrm{EtCO}_{2}$ puede no ser óptima en los prematuros pequeños con enfermedades pulmonares heterogéneas, por lo que la medición de $\mathrm{PaCO}_{2}$ en estos pacientes es imperativa. 


\section{Monitorización de la función cerebral}

El electroencefalograma de amplitud integrada (aEEG) es una técnica neurofisiológica que permite el registro de la actividad eléctrica cerebral generada espontáneamente por las células de la corteza cerebral, a través de electrodos colocados en la superficie de la cabeza del paciente. El aEEG neonatal es uno de los pocos métodos objetivos que explora la integridad funcional de la corteza cerebral inmadura y sus conexiones. ${ }^{14}$

Es necesaria una valoración continua de la función cerebral por medio del aEEG del paciente críticamente enfermo y en el que es imposible realizar un examen neurológico por efecto de la medicación, la ventilación mecánica, etc.

Permite separar tres patrones de actividad eléctrica:

- Normal

- Supresión moderada

- Supresión grave

La evidencia temprana de supresión moderada a grave identifica la evolución neurológica anormal con una sensibilidad del 100\%, VPP de $82 \%$ y VPN de $100 \% .{ }^{15}$ A las 6 horas de vida, el VPP es $86 \%$ y el VPN 91\%.6.,17 Permite el diagnóstico y el seguimiento de las convulsiones subclínicas y su respuesta al tratamiento anticomicial. ${ }^{16}$

Interpretación: al igual que la interpretación del aEEG de los niños mayores, el aEEG del neonato se basa en el conocimiento del patrón normal esperado para cada edad, y la evaluación de la actividad de fondo y de fenómenos transitorios y epileptogénicos. En el RN es fundamental valorar la continuidad del registro, la sincronicidad interhemisférica y los hallazgos mencionados. ${ }^{14}$

Indicaciones: las indicaciones actuales del aEEG comprenden:

- Diagnóstico de disfunciones cerebrales focales o difusas: las anormalidades son inespecíficas, pero pueden aportar evidencias de encefalopatías y de su gravedad.

- Diagnóstico de convulsiones: son descargas rítmicas que duran más de 10 segundos. Las crisis son casi siempre focales, aunque pueden ser multifocales.

- Pronóstico del daño cerebral y episodios convulsivos: un aEEG discontinuo, con intervalos entre descargas mayores de 30 segundos o un aEEG isoeléctrico se asocian con mal pronóstico.

- Otras indicaciones: meningitis, enfermedades metabólicas, hipoglucemia, etc.
- Durante la ECMO el paciente está sedado y sufre variaciones en el flujo sanguíneo cerebral y también convulsiones.

\section{Claves para una correcta monitorización:}

El aEEG es un complemento a la evaluación clínica. Es necesario el entrenamiento del equipo médico para valorar el registro.

\section{Monitorización adecuada del recién nacido según los niveles de complejidad}

Nivel de monitorización necesario para cada sector de internación

Traslado:

- Durante el traslado, ya sea intrahospitalario o extrahospitalario, el paciente debe permanecer monitorizado de la misma forma como se encuentra en la unidad, ya que el traslado supone la continuación del tratamiento instituido, con un riesgo de complicaciones igual o mayor al anterior.

- Es aconsejable mantener la unidad de traslado con un monitor multiparamétrico (MMP), con las características recomendadas para la portabilidad del monitor (Tabla 2). La batería interna debe tener una autonomía mínima de 2 horas, para continuar su carga en la ambulancia, la cual deberá tener enchufes adecuados para monitores, incubadora, respirador, etc.

- Si en la unidad el paciente es de baja complejidad y solo se monitoriza con oxímetro de pulso, se pueden seleccionar las opciones de saturometría y medición de frecuencia cardíaca por pulso en el MMP. En caso de no contar con MMP, se puede reemplazar por un oxímetro de pulso.

- Es recomendable disponer de un MMP destinado específicamente al traslado, que esté fijado a la incubadora de transporte para evitar accidentes y que cuente con mantenimiento preventivo (Tabla 2).

Sala de recepción del $R N$ :

- El monitor requerido para este sector es el saturómetro u oxímetro de pulso.

- En la sala de recepción se debe contar con un saturómetro por cada cuna radiante, para asegurar que cada RN tenga un monitor disponible. La cantidad de cunas radiantes disponibles con su monitor dependerá del número de partos de la unidad.

Es recomendable que cada institución cuente con la tabla de saturación normal en el $\mathrm{RN}$ a los 3, 5 y 10 minutos de vida. 
Sector de internación conjunta:

- Si la política de la institución es realizar tratamientos como antibióticos intravenosos o luminoterapia en el sector de internación conjunta, se recomienda la monitorización con saturómetro hasta el alta.

Sector de bajo riesgo:

- Se considera en este sector a aquellos RN de peso mayor o igual a $2300 \mathrm{~g}$ y en la cuna.

- Los diagnósticos más frecuentes en este nivel de complejidad son: pacientes en buen estado que requieren tratamientos como: luminoterapia, antibioticoterapia intravenosa, internaciones por causa social o enfermedades maternas, pacientes en el momento previo al alta, etc.

- Todos los pacientes en este sector deben estar monitorizados mínimamente con un monitor de FC e idealmente con un saturómetro por paciente, cualquiera que sea la causa.

- La supervisión directa del personal de enfermería no suplanta el requerimiento de monitorización.

- No se recomienda el uso de monitores de apneas como único control.

- En consenso se decide que todos los pacientes ingresados en esta unidad deberán tener mínimamente una medición de tensión

TABLA 2. Características esenciales para el equipamiento del transporte neonatal

- Portátil.

- Duradero, capaz de soportar una fuerza de desaceleración.

- De poco peso.

- De fácil mantenimiento.

- Autonomía de energía (doble del tiempo requerido para el traslado).

- Capacidad AC/DC y conversor para lograr la autonomía.

- Equipos en los que no interfiera la fuerza electromagnética.

- Equipos resistentes a la interferencia electromagnética.

- Pantallas con datos claros, digitales y con forma de onda.

- Bien nivelados.

- Alarmas visibles y audibles, de las variables fisiológicas y de fuentes eléctricas y gases.

- Asegurado con accesorios para que quede adecuadamente fijado.

- Compatible con otros equipos.

- Que cumpla con las normas vigentes de traslado terrestre y aéreo.

- Que tolere cambios de altitud, temperatura, rápida descompresión y vibración.

- Que pase a través de las puertas hospitalarias.

- Que pueda ser trasportado por dos personas. arterial no invasiva durante su internación, independientemente del diagnóstico y de la duración de la internación, entendiéndose que es una oportunidad para detectar las alteraciones de la tensión arterial en el RN.

Sector de cuidados intermedios: se divide en dos sectores:

- Sector de recuperación nutricional: para el RN que ya ha logrado un peso igual o mayor de $1500 \mathrm{~g}$, sin requerimientos de oxígeno. En este caso la monitorización mínima recomendada es el oxímetro de pulso.

- Sector de cuidados intermedios: para el RN menor de $1500 \mathrm{~g}$, con requerimientos de oxígeno o planes de hidratación o nutrición parenteral. En este caso la recomendación es el monitor multiparamétrico con mediciones de TANI según necesidad.

Sector de terapia intensiva neonatal:

- En este sector se encuentran los pacientes que requieren una monitorización intensiva de acuerdo con la terapéutica instituida. Cada paciente debe contar con un monitor multiparamétrico, con medición de ECG, FR, saturometría, TANI y TAI.

- Se debe asegurar que cada monitor multiparamétrico de este sector provea TAI.

- La medición del TC $\mathrm{CO}_{2}$ y el monitor de función cerebral podrían ser herramientas útiles en este sector. No se requiere más de un equipo por servicio, ya que son equipos trasladables.

- Es inapropiado que un RN ingresado en cuidados intensivos sea monitorizado exclusivamente con un oxímetro de pulso.

- En el caso de los RN prematuros con un peso igual o menor de $800 \mathrm{~g}$ en los que la colocación de electrodos en la piel pueda ser riesgosa, sugerimos una monitorización cuidadosa por medio de saturómetro y TAI a través del catéter arterial umbilical o TANI en los casos que lo requieran. Después de la primera semana de vida, una vez completada la epitelización de la piel, se monitoriza de acuerdo con la gravedad del paciente.

Consultorio de seguimiento de alto riesgo:

Todos los pacientes egresados de la unidad de neonatología se consideran de alto riesgo, por lo cual cada consultorio deberá contar mínimamente con un saturómetro y medición de TANI. 


\section{Consideraciones para la adquisición de equipamiento}

El nuevo equipamiento para adquirir implica una erogación importante, por lo que contar con toda la información disponible permitirá una mejor elección. Es importante el asesoramiento de profesionales, ingenieros o técnicos, de tecnología médica.

La decisión de elegir por mejor precio es la más conveniente, siempre y cuando el equipo cumpla con la mayoría de las siguientes recomendaciones:

- Tener modalidad neonatal.

- Versatilidad ante situaciones de riesgo del RN.

- Cumplir con la reglamentación de seguridad eléctrica.

- Conocer la existencia o no, y la autonomía de la batería interna.

Las siguientes son algunas consideraciones que sugerimos tener en cuenta en el momento de adquirir un nuevo equipo:

- Cumplir con las necesidades del servicio de acuerdo con:

- Requerimientos de los pacientes y su complejidad.

- Características del espacio físico.

- Tener representación en la Argentina.

- Contar con la aprobación del ANMAT: debe tener constancia del certificado y no certificado en trámite.

- Disponer de información sobre el parque instalado de equipos. Esto permitirá comunicarse con otros usuarios y compartir experiencias.

- Asegurar un buen servicio de posventa.

- Asegurar la disponibilidad de accesorios y repuestos.

- Disponer de un manual en castellano por cada equipo. Estos deben estar a mano en los sectores de internación y accesibles las 24 horas.

- Asegurar el tiempo de garantía óptimo y confirmar en qué momento comienza.

- Analizar si la tecnología que se está por adquirir ya ha sido superada por otra más novedosa.

- Averiguar si existe disponibilidad de equipos de reemplazo en la empresa vendedora.

- Averiguar si existe la posibilidad de actualización del software.

- Asegurar la capacitación de los usuarios y de los técnicos de la institución que adquiere el equipo por parte de la empresa vendedora.

\section{Higiene del equipamiento}

- Leer atentamente las recomendaciones del fabricante sobre la higiene de los equipos.

- La ubicación debe ser accesible para permitir la limpieza y los ajustes.

- No se debe emplear alcohol para su limpieza: quita el brillo de la pantalla y, con el tiempo, se opaca.

- No emplear químicos, povidona yodada ni clorhexidina.

- No obstruir la ventilación del equipo.

- La higiene de los equipos debe realizarse con un trapo húmedo o limpiadores habilitados.

\section{CONCLUSIONES}

Una correcta monitorización permite obtener información esencial para tomar decisiones terapéuticas adecuadas, y también anticipar o detectar precozmente eventos adversos con el objetivo principal de alcanzar un cuidado seguro. Emplear el equipo adecuado según la complejidad de los pacientes constituye un estándar de atención en la práctica clínica y permite a los profesionales médicos y de enfermería brindar cuidados de calidad.

\section{Agradecimientos}

A los revisores de este consenso: Dra. Norma Rossato, Dr. Gonzalo Mariani, Dr. Guillermo Salas, Lic. Graciela Arimany y Lic. Alejandro Miranda.

\section{BIBLIOGRAFÍA}

1. Ceriani Cernadas J. Manual de Procedimientos en Neonatología. Buenos Aires: Ed Panamericana; 2005.

2. Nuntnarumit P, Yang W, Bada-Ellzey HS. Blood pressure measurements in the newborn. Clin Perinatol 1999;26(4):981.

3. Golombek SG, Fariña D, Sola A, Baquero H, et al. Segundo Consenso Clínico de la Sociedad Iberoamericana de Neonatología: manejo hemodinámico del recién nacido. Rev Panam Salud Pública 2011;29(4):281-302.

4. MacDonald Mhairi G, RamasethuJ K. Atlas of Procedures in Neonatology, $4^{\text {th }}$ Ed. Lippincott: Williams \& Wilkins; 2007.

5. Fernández Colomer R. Grupo de Hospitales Castrillo. Estudioprospectivo sobre el empleo de catéteres umbilicales en el recién nacido. An Pediatr (Barc) 2000;53:470-8.

6. Barrington KL. Umbilical catheters in the newborn: effects of positions of catheters tips. Cochrane Database Syst Rev 2000;2:CD00505.

7. Ramasethu J. Complications of vascular catheters in the Neonatal Intensive Care Unit. Clin Perinatol 2008;35(1):199222.

8. Boo NY, Wong NC, Zulkiffi SS, Lye MS, et al. Risk factors associated with umbilical vascular catheters associated thrombosis in newborn infants. J Paediatr Child Health 1999;35:460-5. 
9. Eberhard P. The design, use, and results of transcutaneous carbon dioxide analysis: current and future directions. Anesth Analg 2007;105:S48-52.

10. Tobias JD. Transcutaneous carbon dioxide monitoring in infants and children. Paediatr Anaesth 2009;19(5):434-44.

11. Fuke S, Miyamoto K, Ohira H, Ohira M, et al. Evaluation of transcutaneous $\mathrm{CO}_{2}$ responses following acute changes in $\mathrm{PaCO}_{2}$ in healthy subjects. Respirology 2009;14(3):436-42.

12. Sivarajan VB, BohnD. Monitoring of standardhemodynamic parameters: heart rate, systemic blood pressure, atrial pressure, pulse oximetry, and end-tidal $\mathrm{CO}_{2}$. Pediatr Crit Care Med 2011;12(4 Suppl):S2-S11.

13. Schmölzer GM, Poulton DA, Dawson JA, Kamlin CO, et al. Assessment of flow waves and colorimetric $\mathrm{CO}_{2}$ detector for endotracheal tube placement during neonatal resuscitation. Resuscitation 2011;82(3):307-12.
14. Hellstrom-Westas L, Rose'n I,Svenningsen NW. Predictive value of early continuous amplitude integrated EEG recordings on outcome after severe birth asphyxia in full term infants. Arch Dis Child 1995;72:F34e8.

15. Al Naqeeb N, Edwards AD, Cowan FM, Azzopardi D. Assessment of neonatal encefalopathy by amplitudeintegrated electroencephalography. Pediatrics 1999;103:1263-71.

16. Toet MC, Hellström-Westas L, Groenendaal F, Eken P, de Vries LS. Amplitude integrated EEG at 3 and 6 hours after birth in full term neonates with hypoxic ischaemic encephalopathy. Arch Dis Child 1999;81:F19e23.

17. Pressler RM, Boylan GB, Morton M, Binnie CD, Rennie JM. Early serial EEG in hypoxic ischemic encephalopathy. Clin Neurophysiol 2001;112: 31-7. 